\title{
Daunorubicin/Cytarabine Liposome: A Review in Acute Myeloid Leukaemia
}

\author{
Hannah A. Blair ${ }^{1}$ \\ Published online: 3 December 2018 \\ ๑) Springer Nature 2018, corrected publication 2018
}

\begin{abstract}
$\operatorname{VYXEOS}^{\mathrm{TM}}$ is a liposomal-encapsulated formulation of daunorubicin and cytarabine delivering a fixed, synergistic 1:5 molar ratio (hereafter referred to as daunorubicin/cytarabine liposome). Daunorubicin/cytarabine liposome is approved in several countries worldwide for the treatment of adults with therapy-related acute myeloid leukaemia (tAML) and AML with myelodysplasia-related changes (MRC). Approval was based on its clinical benefit in older patients with newly diagnosed high-risk/secondary AML in a pivotal phase III trial. In this study, daunorubicin/cytarabine liposome significantly prolonged overall survival (OS) and event-free survival (EFS) relative to conventional chemotherapy with cytarabine plus daunorubicin (hereafter referred to as $7+3$ ). Daunorubicin/cytarabine liposome was also associated with significantly higher rates of complete remission (CR) and CR with incomplete haematological recovery (CRi) compared with 7+3. Daunorubicin/ cytarabine liposome had an acceptable tolerability profile in older patients with newly diagnosed high-risk/secondary AML. The safety profile of daunorubicin/cytarabine liposome, including types and severities of adverse events, was generally similar to that of $7+3$. Therefore, daunorubicin/cytarabine liposome is an important treatment option for adults with newly diagnosed tAML or AML-MRC.
\end{abstract}

Daunorubicin/cytarabine liposome: clinical considerations in acute myeloid leukaemia

Liposomal formulation of daunorubicin and cytarabine in a fixed 1:5 molar ratio

Significantly prolongs OS and EFS relative to $7+3$ chemotherapy in older patients with newly diagnosed high-risk/secondary AML

Associated with significantly higher rates of CR and CRi relative to $7+3$ chemotherapy

Acceptable tolerability profile

The manuscript was reviewed by: $\boldsymbol{B}$. W. Cooper, Division of Hematology and Oncology, Case Western Reserve University and University Hospitals, Cleveland Medical Center, Cleveland, OH, USA; L. E. Damon, Division of Hematology/Oncology, University of California, San Francisco, CA, USA.

Hannah A. Blair

demail@springer.com

1 Springer, Private Bag 65901, Mairangi Bay, Auckland 0754, New Zealand

\section{Introduction}

Acute myeloid leukaemia (AML) is a heterogeneous haematological malignancy characterized by the expansion of myeloid cells in the blood and bone marrow [1]. It is the most common type of acute leukaemia in adults. The incidence of AML increases with age; the median age at diagnosis is 67 years [1]. Secondary AML, including therapyrelated AML (tAML) and AML with myelodysplasia-related changes (MRC), is associated with poorer clinical outcomes than de novo AML [2-5].

For several decades, conventional induction regimens for AML in patients aged $<60$ years have consisted of a continuous 7-day infusion of cytarabine plus an anthracycline (e.g. daunorubicin) on days $1-3$ (hereafter referred to as $7+3$ ) [1]. However, remission rates and survival with conventional induction therapy are lower in older patients ( $\geq 60$ years) compared with younger patients with AML $[3,5]$. Efforts to improve the efficacy of the cytarabine plus daunorubicin backbone (via administration of high-dose cytarabine, anthracycline dose intensification and the addition of various agents) have largely failed to improve outcomes $[3,5,6]$.

$\mathrm{VYXEOS}^{\mathrm{TM}}$ is a liposomal formulation of daunorubicin and cytarabine in a fixed 1:5 molar ratio (hereafter referred 
to as daunorubicin/cytarabine liposome). It is approved in several countries, including the USA [7] and those of the EU [8], for the treatment of adults with newly diagnosed tAML or AML-MRC. This article reviews pharmacological, clinical efficacy and tolerability data relevant to the use of daunorubicin/cytarabine liposome in this setting.

\section{Pharmacodynamic Properties of Daunorubicin/Cytarabine Liposome}

Daunorubicin/cytarabine liposome is a fixed combination of the anthracycline topoisomerase inhibitor daunorubicin and the nucleoside metabolic inhibitor cytarabine, encapsulated in liposomes [7]. The 1:5 molar ratio of daunorubicin/ cytarabine liposome yields maximally synergistic and minimally antagonistic antitumour activity in vitro and in vivo [9, 10]. Daunorubicin exhibits antimitotic and cytotoxic activity through several mechanisms, including the formation of complexes with DNA, inhibition of topoisomerase II activity, inhibition of DNA polymerase activity, regulation of gene expression and production of DNA-damaging free radicals $[7,8]$. Cytarabine affects cells only during the S-phase of cell division and acts primarily through inhibition of DNA polymerase [7, 8]. Daunorubicin/cytarabine liposomes enter the bone marrow and are taken up intact by leukaemia cells [7, 8, 11]. Following cellular internalization, encapsulated daunorubicin/cytarabine is released intracellularly and accumulates in the nucleus [11].

Daunorubicin/cytarabine liposome, compared with the same molar ratio of free cytarabine and daunorubicin, showed enhanced selective in vitro cytotoxicity for AML blasts over normal peripheral blood mononuclear cells [12]. Similarly, in a murine model of leukaemia, daunorubicin/ cytarabine liposomes accumulated in the bone marrow and were selectively taken up to a greater extent by leukaemia cells than by normal bone marrow cells [11]. Scavenger receptor class B type I, which is highly expressed in some human cancers (including myeloid leukaemia) and is able to bind to negatively charged liposomes, may facilitate the uptake of daunorubicin/cytarabine liposome into leukaemia cells [13]. The ex vivo cytotoxicity of daunorubicin/cytarabine liposome was increased for AML blasts exhibiting the FLT3 internal tandem duplication phenotype [14]. This finding suggests that daunorubicin/cytarabine liposome may particularly benefit AML patients with high-risk characteristics that are typically associated with poor prognosis, including specific phenotypes/genotypes [14].

The antitumour effects of daunorubicin/cytarabine liposome have been observed in early-phase clinical trials in patients with AML or high-risk myelodysplasia [15-17]. In a phase 1 dose-escalation study $(n=48)$, daunorubicin/ cytarabine liposome was well tolerated with strong signs of clinical activity when administered at doses of 3-134 units/ $\mathrm{m}^{2}$ on days 1,3 and 5 [15]. The maximum tolerated dose of daunorubicin/cytarabine liposome was 101 units $/ \mathrm{m}^{2}$ [15]; thus, a dose of 100 units $/ \mathrm{m}^{2}$ (delivering daunorubicin $44 \mathrm{mg} /$ $\mathrm{m}^{2}$ and cytarabine $100 \mathrm{mg} / \mathrm{m}^{2}$ ) was subsequently taken forward into phase II/III trials (Sect. 4) [18].

Daunorubicin/cytarabine liposome 100 units $/ \mathrm{m}^{2}$ does not cause clinically relevant QT interval prolongation [19]. At $4 \mathrm{~h}$ post-dose on day 1, the largest absolute and mean changes from time-matched baseline in QTcF were 12.56 and $8.03 \mathrm{~ms}[19]$.

\section{Pharmacokinetic Properties of Daunorubicin/Cytarabine Liposome}

In patients with AML or high-risk myelodysplasia, daunorubicin/cytarabine liposome demonstrated mono-exponential, first-order elimination with minimal early-phase distribution $[15,17]$. The synergistic $1: 5$ molar ratio was maintained in plasma for $>24 \mathrm{~h}$ at all dose levels $[15,17]$. When administered as the fixed combination, daunorubicin and cytarabine exhibited linear single-dose (day 1) and multiple-dose (day 5) pharmacokinetics in terms of maximum plasma concentration and area under the plasma concentration-time curve (AUC) from time 0 to the end of the dosing interval [15]. Across a dose range of daunorubicin/cytarabine liposome $1.3 / 3-59 / 134 \mathrm{mg} / \mathrm{m}^{2}$ (i.e. $0.03-1.3$ times the recommended dosage), there was no evidence of time-dependent kinetics or major departures from dose-proportionality [7, 8]. The accumulation ratio is 1.3 for daunorubicin and 1.4 for cytarabine $[7,8]$.

Both daunorubicin and cytarabine are extensively metabolized after being released from the liposomes [8]. Daunorubicin is catalyzed by aldo-keto reductase and carbonyl reductase enzymes to the active metabolite daunorubicinol, while cytarabine is metabolized by cytidine deaminase to the inactive metabolite 1- $\beta$-D-arabinofuranosylracil (AraU) $[7,8]$. Following administration of daunorubicin/cytarabine liposome $44 / 100 \mathrm{mg} / \mathrm{m}^{2}$ on days 1,3 and 5 , the mean AUC last metabolite:parent ratios for daunorubicinol and AraU were 1.79 and $3.22 \%$ of those for daunorubicin and cytarabine [8].

Urinary excretion of daunorubicin and daunorubicinol accounts for $9 \%$ of the administered dose of daunorubicin, while urinary excretion of cytarabine and AraU accounts for $71 \%$ of the administered dose of cytarabine [7, 8]. Daunorubicin/cytarabine liposome has a prolonged half-life (31.5 $\mathrm{h}$ for daunorubicin and $40.4 \mathrm{~h}$ for cytarabine), with most (>99\%) of the daunorubicin and cytarabine in the circulation remaining encapsulated within the liposomes. The clearance of daunorubicin and cytarabine is 0.16 and $0.13 \mathrm{~L} / \mathrm{h}[7,8]$. Liposomal daunorubicin and cytarabine 
clearances were highly correlated in plasma $\left(\mathrm{r}^{2}=0.82\right)$, supporting prolonged encapsulation in the liposome [16].

The pharmacokinetics of daunorubicin/cytarabine liposome are not affected to any clinically relevant extent by age, sex, race, bodyweight, body mass index or white blood cell count $[7,8]$. The pharmacokinetics of daunorubicin/cytarabine liposome are not significantly altered in patients with mild [creatinine clearance $\left(\mathrm{CL}_{\mathrm{CR}}\right) 60-89 \mathrm{~mL} / \mathrm{min}$ ] or moderate $\left(\mathrm{CL}_{\mathrm{CR}} 30-59 \mathrm{~mL} / \mathrm{min}\right)$ renal impairment, or in patients with a bilirubin level of $\leq 3 \mathrm{mg} / \mathrm{dL}$ (Sect. 6). The effects of severe renal impairment $\left(\mathrm{CL}_{\mathrm{CR}} 15-29 \mathrm{~mL} / \mathrm{min}\right)$, end-stage renal disease (ESRD) or a bilirubin level of $>3 \mathrm{mg} / \mathrm{dL}$ on the pharmacokinetics of daunorubicin/cytarabine liposome are unknown $[7,8]$.

Formal drug interaction studies with daunorubicin/cytarabine liposome have not been conducted; however, the liposomal formulation is expected to reduce the potential for drug-drug interactions [8]. Cardiac function should be closely monitored when daunorubicin/cytarabine liposome is coadministered with cardiotoxic agents [7, 8]. Likewise, hepatic function should be closely monitored when daunorubicin/cytarabine liposome is coadministered with hepatotoxic agents $[7,8]$.

\section{Therapeutic Efficacy of Daunorubicin/ Cytarabine Liposome}

This section focuses on the efficacy of daunorubicin/cytarabine liposome in older patients with newly diagnosed highrisk/secondary AML, as evaluated in a pivotal, open-label, multicentre, phase III trial [20]. Some data are available as abstracts [21-28]. The efficacy of daunorubicin/cytarabine liposome in older adults with newly diagnosed AML (including secondary AML) was initially established in a phase II trial ( $n=125$ evaluable) [18]. The primary endpoint was complete response, defined as complete remission (CR) plus $\mathrm{CR}$ with incomplete haematological recovery (CRi). In this trial, daunorubicin/cytarabine liposome was associated with a higher response rate than conventional $7+3$ chemotherapy with cytarabine plus daunorubicin (67 vs. 51 ; $p=0.07)$, meeting predefined criteria for success $(p<0.1)$. A planned subgroup analysis in patients with secondary AML $(n=52)$ demonstrated an improved response rate with daunorubicin/cytarabine liposome versus $7+3$ (58 vs. $32 \%$; $p=0.06$ ), providing rationale for the subsequent phase III trial [18].

The phase III trial enrolled patients aged 60-75 years with newly diagnosed tAML $(n=63)$, AML with antecedent myelodysplastic syndrome (MDS; $n=145$ ) or chronic myelomonocytic leukaemia (CMML; $n=23$ ), or de novo AML with MDS-related cytogenetic abnormalities $(n=78)$
[20]. Patients who had been previously treated with hypomethylating agents were eligible for inclusion [20].

After stratification by age (60-69 vs. $70-75$ years) and AML type, patients were randomized to receive one or two induction cycles of intravenous daunorubicin/cytarabine liposome $(n=153)$ or $7+3$ chemotherapy $(n=156)$, followed by (post-remission) consolidation therapy with a similar regimen [20]. The initial daunorubicin/cytarabine liposome induction cycle consisted of $44 / 100 \mathrm{mg} / \mathrm{m}^{2}$ administered as a 90-min infusion on days 1, 3 and 5; patients who did not achieve hypoplastic marrow on day 14 received a second induction cycle (at the same dose) on days 1 and 3. Patients who achieved CR/CRi after induction therapy received up to two consolidation cycles consisting of daunorubicin/cytarabine liposome $29 / 65 \mathrm{mg} / \mathrm{m}^{2}$ on days 1 and 3 . The initial $7+3$ induction course consisted of cytarabine $100 \mathrm{mg} / \mathrm{m}^{2} /$ day administered as a 7 -day continuous infusion with daunorubicin $60 \mathrm{mg} / \mathrm{m}^{2}$ on days $1-3$. The second induction cycle and consolidation cycles consisted of cytarabine $100 \mathrm{mg} / \mathrm{m}^{2} /$ day administered as a 5 -day continuous infusion with daunorubicin $60 \mathrm{mg} / \mathrm{m}^{2}$ on days 1 and 2 [20].

The mean age of patients at baseline was 68 years and most $(61 \%)$ patients were male [20]. Patients had an Eastern Cooperative Oncology Group (ECOG) performance status of $0(n=82), 1(n=190)$ or $2(n=37)$. Cytogenetic risk was assessed in 289 patients and was rated as favourable $(n=12)$, intermediate $(n=122)$ or unfavourable $(n=155)$. The primary endpoint was overall survival (OS). The survival analysis was conducted when 236 deaths had occurred (median follow-up of 20.7 months). Efficacy analyses were conducted in the intention-to-treat population [20].

\subsection{Overall Survival}

Daunorubicin/cytarabine liposome significantly improved OS relative to conventional $7+3$ chemotherapy in older adults with newly diagnosed high-risk/secondary AML [20]. Median OS was prolonged by 3.61 months in daunorubicin/cytarabine liposome compared with $7+3$ recipients (Table 1), corresponding to a significant $31 \%$ reduction in the risk of death. According to a multivariate analysis, baseline factors associated with improved OS included lower ECOG performance status $(p=0.034)$, non-adverse karyotype $(p<0.001)$, platelet count $>50 \times 10^{9} / \mathrm{L}(p<0.001)$, white blood cell count $<20 \times 10^{9} / \mathrm{L}(p=0.041)$ and daunorubicin/cytarabine liposome treatment group $(p=0.008)$. Kaplan-Meier estimates of OS at 1 and 2 years are shown in Table 1 [20]. The number needed to treat to prevent 1 death at 2 years with daunorubicin/cytarabine liposome versus $7+3$ was 6 [28].

Prespecified and post hoc subgroup analyses demonstrated that the efficacy of daunorubicin/cytarabine liposome was generally consistent across a broad patient population 
Table 1 Efficacy of daunorubicin/cytarabine liposome in older patients with newly diagnosed high-risk/secondary acute myeloid leukaemia in a randomized, multicentre, phase III trial

\begin{tabular}{llllllllll} 
Treatment [20] & No. of pts & Median OS & 1-year OS & 2-year OS & CR/CRi & CR & $\begin{array}{l}\text { Median } \\
\text { remission } \\
\text { duration }\end{array}$ & Median EFS $^{b}$ \\
\cline { 3 - 9 } & & & & & & & & \\
& & Months (HR; 95\% CI) & $\%$ of pts & $\%$ of pts & $\%$ of pts & $\%$ of pts & Months & Months (HR; 95\% CI) \\
\hline DNR/CYT & 153 & $9.56(0.69 ; 0.52-0.90) * *$ & 42 & 31 & $48^{*}$ & $37 *$ & 6.93 & $2.53(0.74 ; 0.58-0.96)^{*}$ \\
\hline $7+3$ & 156 & 5.95 & 28 & 12 & 33 & 26 & 6.11 & 1.31 \\
\hline
\end{tabular}

$7+3$ conventional cytarabine + daunorubicin chemotherapy, $C R$ complete remission, $C R i$ CR with incomplete haematological recovery, DNR/ $C Y T$ daunorubicin/cytarabine liposome, $E F S$ event-free survival, $H R$ hazard ratio (DNR/CYT vs. $7+3$ ), $O S$ overall survival, $p t s$ patients

${ }^{*} p<0.05,{ }^{*} p=0.003$ vs. $7+3$ ( $p$ value for OS is one-sided; all other $p$ values are two-sided

${ }^{\text {a}}$ Primary endpoint

${ }^{\mathrm{b}}$ Time from randomization to date of induction failure, relapse from CR/CRi or death from any cause

[20-26]. Daunorubicin/cytarabine liposome significantly improved OS versus $7+3$, irrespective of age (60-69 vs. $70-75$ years) [20] and in patients with refractory anaemia with excess blasts in transformation (RAEB-t) [22], tAML [23], AML with antecedent MDS or CMML [20], wildtype FLT3 [20] and favourable/intermediate cytogenetic risk [20]. Median OS was numerically longer with daunorubicin/cytarabine liposome versus $7+3$, regardless of whether the first cycle of consolidation was administered in an inpatient (14.72 vs. 9.26 months) or outpatient (25.43 vs. 6.87 months) setting [25]. Median OS was longer with daunorubicin/cytarabine liposome versus $7+3 / 5+2$ in patients who received consolidation (25.43 vs. 8.53 months), regardless of whether they received consolidation followed by transplantation (not reached vs. 9.82 months) or consolidation without transplantation (13.67 vs. 8.44 months) [26].

In a pooled analysis of data from the phase II [18] and III [20] trials $(n=353)$, daunorubicin/cytarabine liposome significantly improved OS compared with $7+3$ (9.63 vs. 5.59 months; HR 0.64; 95\% CI 0.49-0.83) [21]. OS at 1 year was $42 \%$ for daunorubicin/cytarabine liposome and $27 \%$ for $7+3[21]$.

\subsection{Other Endpoints}

Remission rates (CR, CR/CRi) were significantly greater in daunorubicin/cytarabine liposome than $7+3$ recipients; however, there were no significant between-group differences in the median duration of remission (Table 1) [20]. $\mathrm{CR} / \mathrm{CRi}$ rates favoured daunorubicin/cytarabine liposome over $7+3$ across age groups and AML subtypes [20]. Patients treated with daunorubicin/cytarabine liposome were more likely to achieve remission after one induction cycle than those treated with $7+3$, both for CR [ 45 vs. $28 \%$; odds ratio (OR) 2.08; 95\% CI 1.17-3.73] and CR/CRi (55 vs. 34\%; OR 2.40; 95\% CI 1.36-4.21) [27]. Remission rates after two induction cycles were similar between treatment groups [27].

Among patients who achieved CR/CRi, the cumulative incidence function estimates for relapse at 6 months and 1 year were 0.35 and 0.67 in the daunorubicin/cytarabine liposome group versus 0.43 and 0.63 in the $7+3$ group (events included relapse from CR/CRi and death) [20]. The rate of relapse at 1 year among patients with CR/CRi was $26 \%$ in the daunorubicin/cytarabine liposome group and $27 \%$ in the $7+3$ group [20].

Median event-free survival was significantly longer with daunorubicin/cytarabine liposome than with $7+3$ (Table 1) [20]. The rate of allogeneic haematopoietic cell transplant (HCT) was 34\% in the daunorubicin/cytarabine liposome group and $25 \%$ in the $7+3$ group; the majority of patients proceeding to HCT were in $\mathrm{CR}$ or $\mathrm{CRi}$. An exploratory landmark post-HCT survival analysis favoured daunorubicin/ cytarabine liposome over 7+3 (HR 0.46; 95\% CI 0.24-0.89; $p=0.009$ ). Early mortality rates with daunorubicin/cytarabine liposome and $7+3$ were 6 and $11 \%$ at day 30, and 14 and $21 \%$ at day 60 [20].

\section{Tolerability of Daunorubicin/Cytarabine Liposome}

Intravenous daunorubicin/cytarabine liposome had an acceptable tolerability profile in older patients with newly diagnosed high-risk/secondary AML in the pivotal phase III trial discussed in Sect. 4 [20]. The safety profile of daunorubicin/cytarabine liposome, including types and severities of adverse events (AEs), was similar to that of conventional $7+3$ chemotherapy [20].

During the induction phase, the AEs of any grade that occurred most frequently ( $\geq 40 \%$ incidence) with daunorubicin/cytarabine liposome versus $7+3$ were haemorrhage (70 vs. $49 \%$ ), febrile neutropenia (68 vs. $68 \%$ ), rash (54 vs. 
$36 \%$ ), oedema (51 vs. $60 \%$ ), nausea (47 vs. $52 \%$ ), diarrhoea/ colitis (45 vs. $66 \%$ ), mucositis (44 vs. $46 \%$ ) and constipation (40 vs. $38 \%$ ) [7]. The most common ( $\geq 20 \%$ incidence) grade 3-5 AEs during induction therapy were febrile neutropenia (66 vs. $68 \%$ ), bacteraemia ( 23 vs. $21 \%$ ) and pneumonia (20 vs. 17\%). During the consolidation phase (both cycles pooled), the most common AEs in the daunorubicin/cytarabine liposome group were haemorrhage (43\%) and febrile neutropenia (29\%). Treatment discontinuation due to AEs occurred in 18 and $13 \%$ of daunorubicin/cytarabine liposome and $7+3$ recipients. AEs leading to discontinuation of daunorubicin/cytarabine liposome included cytopenias, infection, cardiotoxicity, respiratory failure, haemorrhage, renal insufficiency and colitis. Fatal AEs (not in the setting of progressive disease) occurred in $6 \%$ of patients in each treatment group; these included infection, CNS haemorrhage and respiratory failure [7].

Patients in the daunorubicin/cytarabine liposome group had an $\approx 50 \%$ longer median duration of treatment (and therefore a longer period of $\mathrm{AE}$ reporting) than those in the $7+3$ group [29]. The median rate of AEs (reported during or within 30 days of the end of treatment) per patient-year was 75.68 with daunorubicin/cytarabine liposome and 87.22 with $7+3$. Corresponding median rates of grade $\geq 3$ AEs were 12.24 and 13.53 per patient-year. The median rate of serious AEs per patient-year was 3.15 with daunorubicin/ cytarabine liposome and 0.0 with $7+3$; this may have been partly due to more daunorubicin/cytarabine liposome than $7+3$ recipients receiving consolidation therapy in an outpatient setting (51 vs. $6 \%$ in cycle 1 and 61 vs. $0 \%$ in cycle 2 ) and potentially requiring hospitalization to manage their (serious) AEs [29].

There have been reports of severe myelosuppression (including fatal infections and haemorrhagic events) with daunorubicin/cytarabine liposome [8]. Serious or fatal haemorrhages (including fatal CNS haemorrhages) associated with prolonged severe thrombocytopenia have been reported in patients receiving daunorubicin/cytarabine liposome [7, 8]. Among patients receiving daunorubicin/cytarabine liposome in clinical trials $(n=375)$, the incidence of infections due to neutropenia (e.g. pneumonia, sepsis and bacteraemia) was $78 \%$ and the incidence of haemorrhagic events was $69 \%$ [8]. In the phase III trial, the median time to recovery from neutropenia [absolute neutrophil count (ANC) $\geq 500 /$ $\mu \mathrm{L}]$ and thrombocytopenia (platelet count $\geq 50,000 / \mu \mathrm{L}$ ) in patients who achieved CR/CRi after initial induction therapy was longer with daunorubicin/cytarabine liposome (35 and 37 days) than with $7+3$ (29 and 29 days) [20]. Similar results were seen in patients who achieved CR/CRi after the second induction cycle ( 35 and 35 vs. 28 and 24 days, respectively). Infection-related events of any grade occurred in 93\% of patients in both treatment groups. The incidence of grade 3-5 infection was $84 \%$ with daunorubicin/cytarabine liposome and $86 \%$ with $7+3$; corresponding rates of grade 5 infection were 7 and 3\%. The incidence of bleeding events of any grade was $75 \%$ with daunorubicin/cytarabine liposome and $60 \%$ with $7+3$; corresponding rates of grade $3-5$ bleeding events were 12 and $9 \%$. Grade 5 bleeding-related events occurred in 3\% of patients in both treatment groups [20]. Supportive measures (e.g. anti-infectives, colony-stimulating factors, transfusions) should be used in patients who develop myelosuppressive complications [8] and blood counts should be regularly monitored until recovery $[7,8]$.

Anthracyclines such as daunorubicin are associated with an increased risk of cardiotoxicity (including congestive heart failure), particularly in patients with pre-existing cardiac disease, previous radiotherapy to the mediastinum, prior therapy with anthracyclines or concomitant use of cardiotoxic drugs [7, 8]. Cardiotoxicity events [e.g. decreased left ventricular ejection fraction (LVEF) and congestive heart failure] were reported in $72 \%$ of daunorubicin/cytarabine liposome recipients in clinical trials; the incidence of serious cardiotoxicity events was 9\% [8]. Cardiac function should be assessed prior to initiation of daunorubicin/cytarabine liposome [7, 8]. Treatment with daunorubicin/cytarabine liposome is not recommended in patients with decreased LVEF or in patients whose lifetime cumulative anthracycline exposure has reached the maximum limit [7]. Daunorubicin/ cytarabine liposome should be discontinued in patients with impaired cardiac function unless the benefits of treatment outweigh the risks $[7,8]$.

There have been reports of serious or fatal hypersensitivity reactions (including anaphylaxis) with daunorubicin and cytarabine [7, 8]. Hypersensitivity reactions were reported in $67 \%$ of daunorubicin/cytarabine liposome recipients in clinical trials, most of which were not serious [8]. In patients who experience a hypersensitivity reaction, treatment interruption, slowing of the infusion rate, symptom management, monitoring and/or permanent discontinuation of daunorubicin/cytarabine liposome may be required depending on the severity of the reaction $[7,8]$.

Reconstituted daunorubicin/cytarabine liposome contains copper [7,8], which is required to retain both drugs inside the liposomes and to modulate their synergistic release (Sects. 2, 3) [30]. When administered as recommended (Sect. 6), the maximum theoretical total copper exposure is $106 \mathrm{mg} / \mathrm{m}^{2}$ [7]. Daunorubicin/cytarabine liposome should only be used in patients with Wilson's disease or other copper-related disorders if the benefits outweigh the risks [7, 8]. In these patients, copper levels should be monitored and serial neuropsychological examinations should be performed [7]. Daunorubicin/cytarabine liposome should be discontinued in patients who experience signs or symptoms of acute copper toxicity $[7,8]$. 


\section{Dosage and Administration of Daunorubicin/Cytarabine Liposome}

In the USA [7] and EU [8], intravenous daunorubicin/cytarabine liposome is indicated for the treatment of adults with newly diagnosed tAML or AML-MRC. The recommended dosage of daunorubicin/cytarabine liposome for the first cycle of induction is $44 / 100 \mathrm{mg} / \mathrm{m}^{2}$ administered as a 90 -min infusion on days 1,3 and $5[7,8]$. If needed, a second cycle of induction therapy (at the same dose) may be administered (2-5 weeks after the first [7]) as a 90-min infusion on days 1 and $3[7,8]$. Consolidation therapy is recommended for patients in remission if the ANC has recovered to $>500 /$ $\mu \mathrm{L}$ and the platelet count has recovered to $>50,000 / \mu \mathrm{L}$, in the absence of unacceptable toxicity [7, 8]. Patients may receive up to two cycles of consolidation, with the first cycle administered 5-8 weeks after the start of the last induction. The recommended dosage of daunorubicin/cytarabine liposome for consolidation is $29 / 65 \mathrm{mg} / \mathrm{m}^{2}$ as a 90 -min infusion on days 1 and 3 [7, 8]. Daunorubicin/cytarabine liposome carries a (boxed [7]) warning stating that it must not be interchanged with other daunorubicin- and/or cytarabinecontaining products $[7,8]$.

The efficacy and tolerability of daunorubicin/cytarabine liposome in paediatric patients has not been established [7, 8]. No dosage adjustment of daunorubicin/cytarabine liposome is required in patients with mild or moderate renal impairment, or in patients with a bilirubin level of $\leq 3 \mathrm{mg} / \mathrm{dL}$ (Sect. 3). Daunorubicin/cytarabine liposome has not been studied in patients with severe renal impairment or ESRD, or in patients with a bilirubin level $>3 \mathrm{mg} / \mathrm{dL}[7,8]$. The EU summary of product characteristics states that daunorubicin/cytarabine liposome should only be used in patients with severe renal or hepatic impairment if the benefits outweigh the risks [8].

Consult local prescribing information for detailed information regarding the dosing schedule, warnings and precautions, drug interactions, management recommendations (i.e. dosage interruptions and discontinuations) for tolerability issues of particular interest and use in special patient populations.

\section{Place of Daunorubicin/Cytarabine Liposome in the Management of Acute Myeloid Leukaemia}

The prognosis of patients with AML is directly related to age, cytogenetics and antecedent myeloid malignancies [2, 5]. There is a need for new treatment approaches for patients who respond poorly to conventional $7+3$ induction therapy, including older patients ( $>60$ years) and patients with secondary AML, including tAML and AML-MRC.
A liposomal-encapsulated formulation of daunorubicin/ cytarabine has been approved in several countries, including the USA [7] and those of the EU [8], for the treatment of adults with newly diagnosed tAML or AML-MRC. The formulation is designed to deliver a fixed 1:5 molar ratio of daunorubicin and cytarabine, which is taken up to a greater extent by leukaemia cells than by normal bone marrow cells (Sect. 2). This preferential uptake, together with prolonged bone marrow exposure to the synergistic drug ratio (Sect. 3), may increase the cytotoxic activity and antileukaemic efficacy of daunorubicin/cytarabine liposome [17]. The significance of this approval is evident in the recently updated NCCN clinical practice guidelines, which recommend the use of daunorubicin/cytarabine liposome as the only category 1 induction treatment option for patients with cytotoxic tAML, antecedent MDS/CMML or cytogenetic changes consistent with MDS [1]. The UK National Institute for Health and Care Excellence recommends daunorubicin/ cytarabine liposome as an option for untreated tAML or AML-MDS in adults [31].

Approval of daunorubicin/cytarabine liposome was based on the findings of a pivotal phase III trial in older patients with newly diagnosed high-risk/secondary AML (Sect. 4). Phase II data $[18,32]$ were used to support an indication in patients with newly diagnosed AML, as well as a limitation to patients with the specified subgroups of AML (i.e. AML-MRC) [33]. Safety data from another phase II trial [32] were used to expand the indication to include all adult patients, rather than restricting it to the older population (60-75 years) enrolled in the phase III trial [33].

In the phase III trial, daunorubicin/cytarabine liposome significantly improved median OS compared with conventional $7+3$ chemotherapy with cytarabine plus daunorubicin (Sect. 4.1). The OS benefits of daunorubicin/cytarabine liposome were generally maintained across age strata and AML subtypes, and were seen in subgroups of patients with RAEB-t, wild-type FLT3 and favourable/intermediate cytogenetic risk. The improved post-HCT survival observed with daunorubicin/cytarabine liposome (Sect. 4.2) suggests that the response may be deeper than that achieved with $7+3$ [20]. Of note, median OS was not diminished when daunorubicin/cytarabine liposome consolidation was administered in the outpatient versus inpatient setting (Sect. 4.1). Moreover, in a small $(n=22)$ pilot study, outpatient induction therapy with daunorubicin/cytarabine liposome was feasible and safe in older patients with newly diagnosed AML [34].

The safety profile of daunorubicin/cytarabine liposome was similar to that of $7+3$ chemotherapy (Sect. 5). However, despite between-group differences in the duration of treatment, daunorubicin/cytarabine liposome was associated with a lower rate of AEs per patient-year than $7+3$. Daunorubicin/cytarabine liposome was associated with slower neutrophil recovery and platelet recovery than $7+3$ (Sect. 5), 
which may be related to prolonged daunorubicin/cytarabine liposome drug exposure in the bone marrow (Sect. 3).

The costs associated with AML constitute a substantial economic burden [35, 36], particularly in older patients who require more frequent hospitalizations and intensive care unit admissions [36]. According to the UK NICE committee, daunorubicin/cytarabine liposome represents a cost-effective use of health system resources for end-of-life treatments [31]. The US Centers for Medicare and Medicaid Services has granted approval for a new technology add-on payment for daunorubicin/cytarabine liposome in adults with newly diagnosed tAML or AML-MRC [37]. The payment will support Medicare beneficiaries' access to daunorubicin/cytarabine liposome treatment in certain inpatient hospital settings [37]. A recent modelled pharmacoeconomic study from a US commercial payer perspective suggested that daunorubicin/cytarabine liposome is a cost effective treatment option for patients with tAML or AML-MRC (abstract data) [38]. A budget-impact model from the perspective of a US healthplan with 1 million members predicted that gradually increasing the use of daunorubicin/ cytarabine liposome would result in cost increases compared with $7+3$ of US $\$ 29,884$ over 3 years, equating to an incremental per-member cost of $<$ US $\$ 0.01$ per month (abstract data) [39]. Further robust pharmacoeconomic analyses would be of interest.

In conclusion, daunorubicin/cytarabine liposome provides a survival benefit with acceptable tolerability in adults with newly diagnosed tAML or AML-MRC. The liposomalencapsulated formulation delivers a synergistic drug ratio and allows for relatively simple administration versus conventional $7+3$ chemotherapy (i.e. a 90-min infusion on days 1, 3 and 5 instead of a continuous 7-day infusion). Daunorubicin/cytarabine liposome is therefore an important treatment option for adults with newly diagnosed tAML or AML-MRC.

\section{Data Selection Daunorubicin/Cytarabine Liposome: 369 records identified}

\section{Duplicates removed}

Excluded during initial screening (e.g. press releases; news reports; not relevant drug/indication; preclinical study; reviews; case reports; not randomized trial)

Excluded during writing (e.g. reviews; duplicate data; small patient number; nonrandomized/phase I/II trials)

Cited efficacy/tolerability articles

Search Strategy: EMBASE, MEDLINE and PubMed from 1946 to present. Clinical trial registries/databases and websites were also searched for relevant data. Key words were cytarabine, daunorubicin, Vyxeos, CPX 351, acute myeloid leukaemia, AML. Records were limited to those in English language. Searches last updated 19 November 2018
Acknowledgements During the peer review process, the manufacturer of daunorubicin/cytarabine liposome was also offered an opportunity to review this article. Changes resulting from comments received were made on the basis of scientific and editorial merit.

\section{Compliance with Ethical Standards}

Funding The preparation of this review was not supported by any external funding.

Conflict of interest Hannah Blair is a salaried employee of Adis/ Springer, is responsible for the article content and declares no relevant conflicts of interest.

Open Access This article is distributed under the terms of the Creative Commons Attribution-NonCommercial 4.0 International License (http://creativecommons.org/licenses/by-nc/4.0/), which permits any noncommercial use, distribution, and reproduction in any medium, provided you give appropriate credit to the original author(s) and the source, provide a link to the Creative Commons license, and indicate if changes were made.

\section{References}

1. National Comprehensive Cancer Network. NCCN clinical practice guidelines in oncology $\left(\mathrm{NCCN}\right.$ guidelines $\left.{ }^{\circledR}\right)$ : acute myeloid leukemia (version 2.2018). 2018. http://www.nccn.org. Accessed 19 Nov 2018.

2. Granfeldt Ostgard LS, Medeiros BC, Sengelov H, et al. Epidemiology and clinical significance of secondary and therapy-related acute myeloid leukemia: a national population-based cohort study. J Clin Oncol. 2015;33(31):3641-9.

3. Chen EC, Fathi AT, Brunner AM. Reformulating acute myeloid leukemia: liposomal cytarabine and daunorubicin (CPX-351) as an emerging therapy for secondary AML. Onco Targets Ther. 2018;11:3425-34.

4. Kayser S, Dohner K, Krauter J, et al. The impact of therapy-related acute myeloid leukemia (AML) on outcome in 2853 adult patients with newly diagnosed AML. Blood. 2011;117(7):2137-45.

5. Boddu PC, Kantarjian HM, Ravandi F, et al. Characteristics and outcomes of older patients with secondary acute myeloid leukemia according to treatment approach. Cancer. 2017;123(16):3050-60.

6. Tolcher AW, Mayer LD. Improving combination cancer therapy: the CombiPlex ${ }^{\circledR}$ development platform. Future Oncol. 2018;14(13):1317-32.

7. Jazz Pharmaceuticals. VYXEOS ${ }^{\mathrm{TM}}$ (daunorubicin and cytarabine) liposome for injection, for intravenous use: US prescribing information. 2017. http://www.fda.gov. Accessed 19 Nov 2018.

8. European Medicines Agency. Vyxeos $44 \mathrm{mg} / 100 \mathrm{mg}$ powder for concentrate for solution for infusion: EU summary of product characteristics. 2018. http://www.ema.europa.eu. Accessed 19 Nov 2018.

9. Mayer LD, Harasym TO, Tardi PG, et al. Ratiometric dosing of anticancer drug combinations: controlling drug ratios after systemic administration regulates therapeutic activity in tumorbearing mice. Mol Cancer Ther. 2006;5(7):1854-63.

10. Tardi P, Johnstone S, Harasym N, et al. In vivo maintenance of synergistic cytarabine:daunorubicin ratios greatly enhances therapeutic efficacy. Leuk Res. 2009;33(1):129-39.

11. Lim WS, Tardi PG, Dos Santos N, et al. Leukemia-selective uptake and cytotoxicity of CPX-351, a synergistic fixed-ratio 
cytarabine: daunorubicin formulation, in bone marrow xenografts. Leuk Res. 2010;34(9):1214-23.

12. Kim HP, Gerhard B, Harasym TO, et al. Liposomal encapsulation of a synergistic molar ratio of cytarabine and daunorubicin enhances selective toxicity for acute myeloid leukemia progenitors as compared to analogous normal hematopoietic cells. Exp Hematol. 2011;39(7):741-50.

13. Di Y, Wasan EK, Cawthray J, et al. Scavenger receptor class BI (SR-BI) mediates uptake of CPX-351 into K562 leukemia cells. Drug Dev Ind Pharm. 2018. https://doi.org/10.1080/03639 045.2018.1513026.

14. Gordon MJ, Tardi P, Loriaux MM, et al. CPX-351 exhibits potent and direct ex vivo cytotoxicity against AML blasts with enhanced efficacy for cells harboring the FLT3-ITD mutation. Leuk Res. 2017:53:39-49.

15. Feldman EJ, Lancet JE, Kolitz JE, et al. First-in-man study of CPX-351: a liposomal carrier containing cytarabine and daunorubicin in a fixed 5:1 molar ratio for the treatment of relapsed and refractory acute myeloid leukemia. J Clin Oncol. 2011;29(8):979-85.

16. Nikanjam M, Capparelli EV, Lancet JE, et al. Persistent cytarabine and daunorubicin exposure after administration of novel liposomal formulation CPX-351: population pharmacokinetic assessment. Cancer Chemother Pharmacol. 2018;81(1):171-8.

17. Feldman EJ, Kolitz JE, Trang JM, et al. Pharmacokinetics of CPX-351; a nano-scale liposomal fixed molar ratio formulation of cytarabine:daunorubicin, in patients with advanced leukemia. Leuk Res. 2012;36(10):1283-9.

18. Lancet JE, Cortes JE, Hogge DE, et al. Phase 2 trial of CPX351 , a fixed 5:1 molar ratio of cytarabine/daunorubicin, vs cytarabine/daunorubicin in older adults with untreated AML. Blood. 2014;123(21):3239-46.

19. Lin TL, Newell LF, Stuart RK, et al. CPX-351 ((cytarabine:daunorubicin) liposome injection, (Vyxeos)) does not prolong QTcF intervals, requires no dose adjustment for impaired renal function and induces high rates of complete remission in acute myeloid leukemia [abstract no. 2510]. Blood. 2015;126(23).

20. Lancet JE, Uy GL, Cortes JE, et al. CPX-351 (cytarabine and daunorubicin) liposome for injection versus conventional cytarabine plus daunorubicin in older patients with newly diagnosed secondary acute myeloid leukemia. J Clin Oncol. 2018;36(26):2684-92.

21. Lancet JE, Ritchie EK, Uy GL, et al. Efficacy and safety of CPX351 versus $7+3$ in older adults with secondary acute myeloid leukemia: combined subgroup analysis of phase 2 and phase 3 studies [abstract no. 2657]. Blood. 2017;130(Suppl 1).

22. Lin TL, Uy GL, Wieduwilt MJ, et al. Subanalysis of patients with secondary acute myeloid leukemia (sAML) with refractory anemia with excess of blasts in transformation (RAEB-T) enrolled in a phase 3 study of CPX-351 versus conventional $7+3$ cytarabine and daunorubicin [abstract no. 1698]. Blood. 2017;130(Suppl 1).

23. Lancet JE, Rizzieri D, Schiller GJ, et al. Overall survival (OS) with CPX-351 versus $7+3$ in older adults with newly diagnosed, therapy-related acute myeloid leukemia (tAML): subgroup analysis of a phase III study [abstract no. 7035]. J Clin Oncol. 2017;35(Suppl 15).

24. Medeiros BC, Hogge D, Newell LF, et al. Overall survival and transplantation in patients with FLT3 mutations: subgroup analysis of a phase 3 study of CPX-351 versus $7+3$ in older adults with newly diagnosed, high-risk acute myeloid leukemia [abstract no. P210]. Haematologica. 2017;102(Suppl 2):53-4.

25. Kolitz JE, Strickland SA, Cortes JE, et al. Efficacy by outpatient vs inpatient administration of consolidation: subgroup analysis of a phase 3 study of CPX-351 versus $7+3$ in older adults with newly diagnosed, high-risk acute myeloid leukemia [abstract no. E922]. Haematologica. 2017;102(Suppl 2):379.

26. Kolitz JE, Strickland SA, Cortes JE, et al. Outcomes in older patients with newly diagnosed, high-risk/secondary acute myeloid leukemia (sAML) who received consolidation in a phase 3 study of CPX-351 versus conventional 7+3/5+2 cytarabine and daunorubicin. Society of Hematologic Oncology Sixth Annual Meeting. 2018.

27. Cortes JE, Medeiros BC, Uy GL, et al. Outcomes by number of induction cycles with CPX-351 versus $7+3$ chemotherapy in older adults with newly diagnosed, high-risk/secondary acute myeloid leukemia (sAML) [abstract no. PF239]. In: 23rd Congress of the European haematology association. 2018.

28. Chung KC, Ryan RJ, Louie AC. CPX-351 for the treatment of newly diagnosed, therapy-related acute myeloid leukemia (tAML) or AML with myelodysplasia-related changes (AML-MRC): an analysis of clinical benefit [abstract no. PCN9]. Value Health. 2018;21(Suppl 1):S15.

29. Medeiros BC, Lancet JE, Cortes JE, et al. Rates of adverse events per patient-year in a randomized, phase 3 study of CPX-351 versus $7+3$ in older adults with newly diagnosed, treatment-related acute myeloid leukemia (AML) or AML with myelodysplasiarelated changes [abstract no. 1366]. Blood. 2017;130(Suppl 1).

30. Dicko A, Kwak S, Frazier AA, et al. Biophysical characterization of a liposomal formulation of cytarabine and daunorubicin. Int $\mathrm{J}$ Pharm. 2010;391:248-59.

31. National Institute for Health and Care Excellence. Liposomal cytarabine-daunorubicin for untreated acute myeloid leukaemia. 2018. http://www.nice.org.uk. Accessed 19 Nov 2018.

32. Cortes JE, Goldberg SL, Feldman EJ, et al. Phase II, multicenter, randomized trial of CPX-351 (cytarabine:daunorubicin) liposome injection versus intensive salvage therapy in adults with first relapse AML. Cancer. 2015;121(2):234-42.

33. US FDA. Vyxeos ${ }^{\circledR}$ (daunorubicin and cytarabine) liposome: multidiscipline review. 2017. http://www.fda.gov. Accessed 19 Nov 2018.

34. Kubal TE, Salamanca C, Komrokji RS, et al. Safety and feasibility of outpatient induction chemotherapy with CPX-351 in selected older adult patients with newly diagnosed AML [abstract no. e19013]. J Clin Oncol. 2018;36(Suppl 15).

35. Hagiwara M, Sharma A, Chung KC, et al. Healthcare resource utilization and costs in patients with newly diagnosed acute myeloid leukemia. J Med Econ. 2018;21(11):1119-30.

36. Sacks NC, Cyr PL, Louie AC, et al. Burden of acute myeloid leukemia among older, newly diagnosed patients: retrospective analysis of data from the 2010-2012 Medicare limited data set. Clin Ther. 2018;40(5):692-703 e2.

37. Jazz Pharmaceuticals. CMS grants new technology add-on payment to Vyxeos ${ }^{\circledR}$ (daunorubicin and cytarabine) liposome for injection [media release]. 3 Aug 2018. http://www.prnewswire .com.

38. Kansal A, Du M, Herrera-Restrepo O, et al. Cost-effectiveness of CPX-351 versus $7+3$ regimen in the treatment of treatmentrelated acute myeloid leukemia (tAML) or AML with myelodysplasia-related changes (MRC) [abstract no. 4674]. Blood. 2017;130(Suppl 1).

39. Jensen IS, Wu E, Sacks N, et al. Budget impact analysis of CPX351 in the treatment of patients with treatment-related acute myeloid leukemia (tAML) or AML with myelodysplasia-related changes (MRC) from a US payer perspective [abstract no. 5615]. Blood. 2017;130(Suppl 1). 